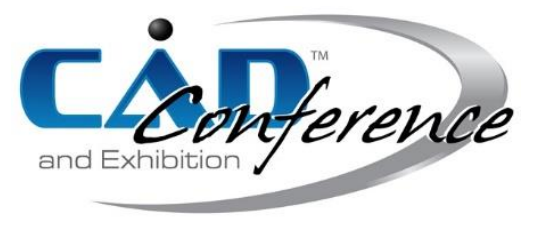

Title:

\title{
Grid Shape Descriptor using Path Integrals for Measuring Sheet Metal Parts Similarity
}

Authors:

Mikko Ylihärsilä, mikko.yliharsila@seamk.fi, Seinäjoki University of Applied Sciences

Juha Hirvonen, juha.hirvonen@seamk.fi, Seinäjoki University of Applied Sciences

Keywords:

Shape descriptor, shape similarity, sheet metal, CAD

DOI: 10.14733/cadconfP.2021.272-276

\section{Introduction:}

Shape matching and similarity measurement are vital for many applications, and they are well-studied subjects in computer science. Most of the studies focus mostly on 2D images rather than 2D CADgeometries. However, similar strategies that are used for images can be applied to the parametric curves used in the CAD-geometries. Shape descriptors can be used both for matching shapes and for measuring similarities.

A shape descriptor is a set of numbers that describe a given shape. It should have some essential properties, such as identifiability, noise resistance, invariance to translation, rotation, scale, affine transform and occultation. Furthermore, the descriptor should be compact to store, and computing the difference between the two descriptors should be simple and efficient. [9]

Shape descriptors can be categorized into three main categories: contour-based descriptors, regionbased descriptors, and hybrid descriptors. The contours-based descriptors use the contour edges for generating the descriptor e.g., by sampling points and generating height histograms. These descriptors are known to be sensitive to noise in the shape and thus not very accurate. However, their computational complexity is low. Region-based descriptors take into account the boundary and the interior of the shape e.g., creating 2D histogram by assigning pixels into 2D bins. And hybrid descriptors combine two or more techniques. [3]

Currently, the most performant descriptor for the MPEG-7 bulls-eye test [4] is a hybrid descriptor [7], which combines discrete cosine transformation (DCT) [6] and height functions [8] based shape descriptors. DCT captures the shape's spatial information, and the height functions capture the boundary of the shape as sampled points.

In this study, a new grid-based shape descriptor is presented, focusing primarily on sheet metal part geometries. Grid-based descriptors can belong to either contour-based or region-based. Early grid-based descriptors were binary-based matrices [2]. Later, the Blurred Shape Model (BSE) [1] was presented, with a matrix containing gray-level values. Most of the descriptors presented earlier are weak in detecting exact duplicates and small differences or they lack the ability to take into account multiple contours and internal shapes. Comparing e.g., two exact duplicate geometries, but the other one rotated can result in lesser or more significant errors depending on the size of the used descriptor vector or sampling frequency.

The descriptor presented here has some similarities to the Blurred Shape Model, but the descriptor is computed for the precise CAD-geometry instead of the rasterized image. The new descriptor can accurately recognize duplicate geometries and distinguish geometries that have slight differences. Most of the other methods use point sampling with a fixed number of points. The new method uses path integrals instead, which notices every small detail in the geometry, which could otherwise fall between 
the sample points when using other methods. The new method also allows search queries for the outer and the inner geometries separately or for the whole part.

\section{Geometry normalization:}

The orientation and the size of the geometry are normalized to allow computing comparable shape descriptor matrices. Geometry normalization is done by computing bounding rectangle around the outer geometry of the part. Geometry is rotated by the bounding rectangle's rotation angle $\boldsymbol{\theta}$ and scaled by inverse of bounding rectangle's width $\boldsymbol{w}$ and height $\boldsymbol{h}$, so that the bounding box of the normalized geometry is a unit square with size of $\mathbf{1} \times \mathbf{1}$.

Bounding box can be computed in many different ways. In this study two methods were used, minimum bounding box and bounding box constructed in the angle of principal axis. These methods align bounding boxes in slightly different angles $\boldsymbol{\theta}$. It is beneficial if the bounding boxes are oriented nearly in the same angle for shapes that are thought to be similar.

\section{Shape descriptor matrix:}

The shape descriptor presented in this study is an $\boldsymbol{n} \times \boldsymbol{n}$ matrix containing positive real numbers. We will call it Smooth Grid Based Shape Descriptor, later referred to as $\boldsymbol{S G B S D}$. SGBSD can be considered as a 2D dimensional grid, where the $(\boldsymbol{n}-\mathbf{2}) \times(\boldsymbol{n}-\mathbf{2})$ innermost cell's overall size is unit square. Therefore, each cell has a size of $\frac{1}{n-2} \times \frac{1}{n-2}$. Scaled geometry will fit inside these innermost cells leaving extra cells outside of the geometry. These extra cells are used to have even weighting everywhere in the geometry. Without the extra cells, the outer geometry would have a smaller impact on the descriptor value.

The value for each element in $\boldsymbol{S G B S D}$ (as seen in equation 1) is calculated by integrating each curve in the geometry over the weight function $\boldsymbol{\omega}(\overrightarrow{\boldsymbol{p}})$ (as seen in equation 2 ). $\boldsymbol{n}_{\boldsymbol{C}}$ is the number of curves. Each curve is defined as parametric curve $\boldsymbol{r}(\boldsymbol{t})$, where $\boldsymbol{t}$ is parameter within interval $[\boldsymbol{a}, \boldsymbol{b}]$. Curve's end points can be calculated when function $\boldsymbol{r}(\boldsymbol{t})$ is evaluated at parameters $\boldsymbol{a}$ and $\boldsymbol{b} \cdot \boldsymbol{r}(\boldsymbol{t})$ is the first derivative of the curve.

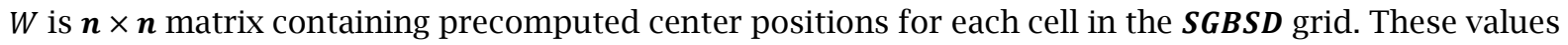
are used to offset the geometry to the corresponding cell being calculated.

Function $\boldsymbol{\omega}(\overrightarrow{\boldsymbol{p}})$ describes the weight of how much the geometry contributes into the cell value in certain position of $\vec{p}$. If the weight function would $\boldsymbol{\omega}(\overrightarrow{\boldsymbol{p}})=\mathbf{1}$, then the integral would result in length of the scaled curve. Parameter $\boldsymbol{\phi}$, which we also refer to as spotsize, defines how far away from the grid cell center point the geometries accumulate the cell value. Each multiple of $\phi$ increases the bottom radius of the weight function by the amount of cell size $\frac{1}{n-2}$. The function is set to zero when the point to be evaluated is further from the cell center point than specified by the parameter $\boldsymbol{\phi}$. Otherwise, the function is a cosine function. The cosine function is modified so that the values are always positive values between 0 to 1 .

$$
\begin{array}{r}
\forall(i, j) \in[n \times n] \operatorname{SGBSD}_{i, j}=\sum_{c=1}^{n_{C}} \int_{a}^{b} \omega\left(r_{c}(t)-W_{i, j}\right) \cdot\left\|\dot{r}_{c}(t)\right\| d t \\
\omega(\vec{p})=\left\{\begin{array}{c}
\frac{\cos \left(\frac{\pi \cdot\|\overrightarrow{ }\|}{\frac{\phi}{n-2}}\right)+1}{2},\|\vec{p}\|<\frac{\phi}{n-2} \\
0,\|\vec{p}\| \geq \frac{\phi}{n-2}
\end{array}\right.
\end{array}
$$

Visualization of this function can be seen in figure 1. The leftmost figure represents rectangular geometry, which is integrated over the weight function $\boldsymbol{\omega}(\overrightarrow{\boldsymbol{p}})$. The integral is easy to understand as surface area of the projected extrusion. The rightmost figure represents the $8 \times 8 \boldsymbol{S G B S D}$ where weight function is plotted for each cell. In this study spotsize of $\boldsymbol{\phi}=\mathbf{1 . 5}$ is used as it gives reasonably uniform weight field over the whole grid. Sum of the weight functions is constant on certain cross-sections, similarly as the sum of the three-phase electricity. However, on 2D-plane it doesn't result in perfect constant sum, but very close. Note that in the figure, only the individual weight functions are plotted, not the sum. 


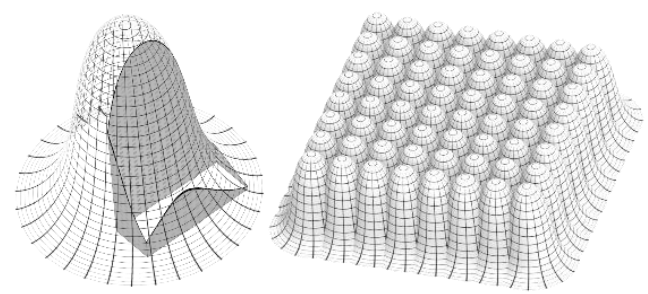

Fig. 1: Left - rectangular geometry integrated over $\omega(\vec{p})$. Right - $8 \times 8$ SGSD weight functions visualized.

Measuring similarity of the shapes:

The length of the shape descriptor is measured using Manhattan distance (as shown in equation 3). Similarly, the difference between the two shape matrices is measured using Manhattan distance (as shown in equation 4). In this study Manhattan distance gave much better results than using Euclidean distance. Previously also Mahalanobis distance has been used instead of Euclidean distance [5].

$$
\begin{gathered}
\operatorname{length}(S G B S D)=\sum_{i=1}^{n} \sum_{j=1}^{n}\left|S G B S D_{i j}\right| \\
\text { difference }\left(S G B S D_{A}, S G B S D_{B}\right)=\sum_{i=1}^{n} \sum_{j=1}^{n}\left|S G B S D_{A_{i j}}-S G B S D_{B_{i j}}\right|
\end{gathered}
$$

The similarity between the two shape descriptor matrices A and B is defined in equation 5 . This equation gives similarity in range 0 to 1 . In the equation, the difference is divided by 2 to avoid negative similarities. The difference can be larger than the maximum length of the shape descriptor. This can be illustrated by thinking of a part that contains one hole. If the hole is removed from the part, it will result in difference that is equal to the sum that the hole contributes to the shape matrix. This would yield to inner geometry similarity of $0 \%$. If the hole is moved into a totally separate location in the part, the difference would be twice from the previous and yield to negative $-100 \%$ similarity. Dividing the difference by 2 will clamp the similarity between 0 and 100\%. Now the previous similarities would be $50 \%$ and $0 \%$. Also using the maximum length as the divider, the order of the parts becomes insignificant and the condition in equation 6 is fulfilled.

$$
\begin{array}{r}
\operatorname{similarity}\left(S G B S D_{A}, S G B S D_{B}\right)=1-\frac{\text { difference }\left(S G S D_{A}, S G S D_{B}\right) / 2}{\max \left(\text { length }\left(S G S D_{A}\right), \operatorname{length}\left(S G S D_{B}\right)\right)} \\
\operatorname{similarity}\left(S G B S D_{A}, S G B S D_{B}\right)=\operatorname{similarity}\left(S G B S D_{B}, S G B S D_{A}\right)
\end{array}
$$

Finally, the shape descriptor matrix A is rotated into four possible angles and the orientation with the maximum similarity is chosen. The same is repeated for the mirrored shape descriptor.

Results:

The practical tests were done with a part database containing 1150 industrial sheet metal parts. Most of the parts were rectangular, containing significant differences in the corner notches. The database contained nearly 100 different part families and many unique parts. Testing was done manually as the dataset was not classified. When querying first 40 unique parts belonging to different part families, the best query matches were every time from the same part family. Approximately $90 \%$ of the parts were more or less rectangular looking shapes, but they contained specific details that made them identifiable to some certain part family group. Unique inner geometry features were queried by drawing a rough sketch of the bounding box of the part and the inner feature to be searched. Figure 2 shows two examples of the correct query results from the database. 


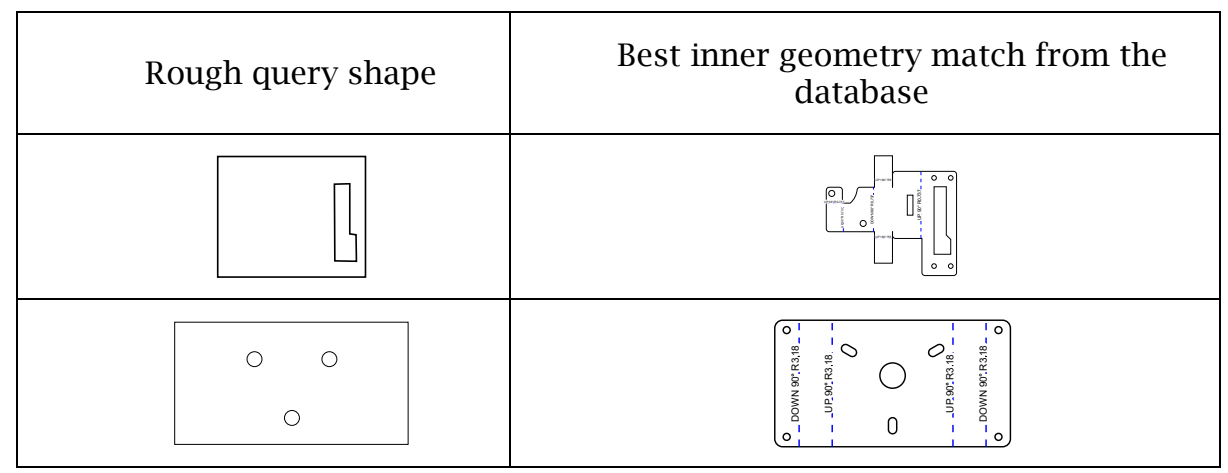

Fig. 2: Searching by inner features.

Duplicate detection performance was tested with a sample part containing a single hole with a diameter of $5 \mathrm{~mm}$ (as seen in figure 3). The part was mirrored and rotated, which should yield identical similarities for both test cases. For both cases, very small errors were measured. The errors can be explained by the numerical noise induced when the geometries were transformed. Then two test cases were done to detect different parts. The hole in the part was moved by $50 \mu \mathrm{m}$ and $1 \mu \mathrm{m}$. The measured errors for these cases were clearly larger, which means that even slight differences in part geometries can be robustly recognized. The scale of the $1 \mu \mathrm{m}$ movement can be illustrated by dividing it by the width of the part, which is $553 \mathrm{~mm}$. $1 \mu \mathrm{m}$ is approximately $0.00018 \%$ of the part's width.

\begin{tabular}{|l|c|c|c|c|}
\hline $\begin{array}{l}\text { Test } \\
\text { case }\end{array}$ & 1 - Original part & 2 - Mirrored part & $\begin{array}{c}3 \text { - Rotated } \\
\text { part }\end{array}$ & $\begin{array}{c}4 \text { - Hole moved } \\
\text { a) } 50 \mu \mathrm{m} \\
\text { b) } 1 \mu \mathrm{m}\end{array}$ \\
\hline Image & & $5.24 \mathrm{e}-15$ & $5.86 \mathrm{e}-15$ & \\
\hline Error & 0.00 & & $\begin{array}{l}\text { a) } 3.59 \mathrm{e}-5 \\
\text { b) } 7.19 \mathrm{e}-7\end{array}$ \\
\hline
\end{tabular}

Fig. 3: Testing duplicate detection robustness.

MPEG-7 database is a well-known benchmark library for comparing shape descriptors [4]. The library contains 1400 images belonging into 70 different classes. A bulls-eye test is performed by counting the correct query results from the first 40 results. This number is then divided by 20 , which is the maximum number of correct matches. The graph on figure 4 shows the bulls-eye scores for 70 different classes. Average performance was $75.1 \%$, which is similar performance as with Blurred Shape Model ( $75 \%$ with 8x8 grid) [1]. For comparison, DCT + height functions hybrid [7], has 91\% bulls-eye score. Our methods performance in MPEG-7 benchmark was mediocre, but the shapes don't present typical industrial sheet metal parts and the performance with these shapes was secondary objective.

Computing shape descriptors for MPEG-7 database took $57.6 \mathrm{~ms} /$ part on average and the pairwise comparison took $0.235 \mathrm{~ms}$ on average using AMD Ryzen 3950X processor. Both computations were executed using a single thread. 


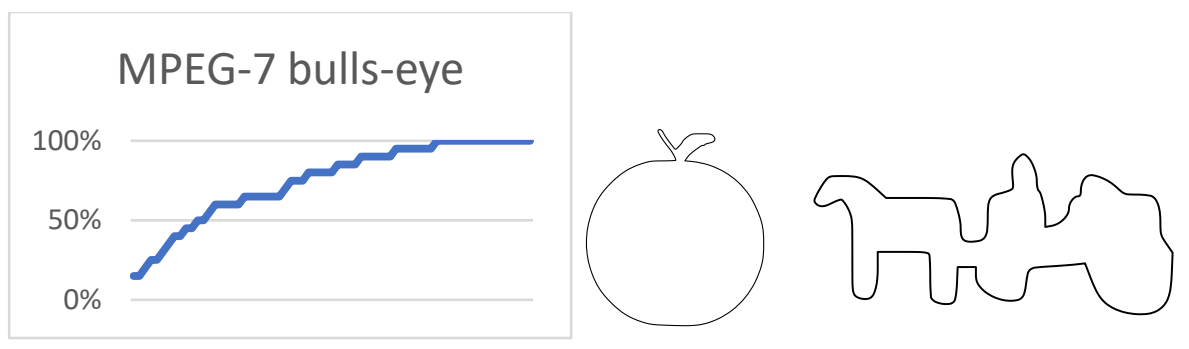

Fig. 4: MPEG-7 bulls-eye performance and two samples.

Conclusions:

The new descriptor's main benefits are the ability to robustly detect duplicates and distinguish almost similar geometries having only a very slight difference. Sheet metal parts belonging to the same part family can have only very slight differences. It is useful to be able to compare them quickly by just comparing the stored shape descriptor values. A new shape descriptor can also be used for searching similar parts according to the outer geometry or the inner geometry separately or for searching for the whole geometry. Searching by inner geometry allows searching for some unique internal shape without knowing the outer geometry details and vice versa.

Performance with generic shapes, e.g., MPEG-7 bulls-eye test, could be improved by combining the presented method with, e.g., height functions [8], which performs better with the single contour geometries having more variety in their shapes in a radial direction. However, for typical sheet metal parts, the benefits of being able to detect small differences and being able to make queries with internal shapes out weighted the property of finding vastly different but for human eyes similar parts.

\section{References:}

[1] Escalera, Sergio; Fornés, Alicia; Pujol, Oriol; Radeva, Petia; Sánchez, Gemma; Lladós, Josep: Blurred Shape Model for binary and grey-level symbol recognition, Pattern Recognition Letters, 30, 2009, 1424-1433, http://doi.org/10.1016/j.patrec.2009.08.001.

[2] Flusser, J: Invariant shape description and measure of object similarity, Proc. 4th International Conference on Image Processing and its Applications, 1992, 139-142.

[3] Kazmi, I. K.; You, L.; Zhang, J. J.: A Survey of 2D and 3D Shape Descriptors, 10th International Conference Computer Graphics, Imaging and Visualization, Macau, 2013, 1-10, http://doi.org/10.1109/CGIV.2013.11.

[4] Latecki, L. J.; Lakamper, R.; Eckhardt T.: Shape descriptors for non-rigid shapes with a single closed contour, Proceedings IEEE Conference on Computer Vision and Pattern Recognition. CVPR 2000 (Cat. No.PR00662), Hilton Head Island, SC, vol.1, 2000, 424-429, http://doi.org/10.1109/CVPR.2000.855850.

[5] Li, Z.; Qu, W.; Cao, J.; Su, Z.; Qi, H.: Geometry-based 2D shape descriptor for retrieval in large database, 2012 IEEE 11th International Conference on Signal Processing, Beijing, 2012, 1096-1101, http://doi.org/10.1109/ICoSP.2012.6491769.

[6] Mannan, A., Babri, H. A.; Saeed, M.: Offline shape recognition using flexible DCT grid, Scientia Iranica, Volume 19, Issue 6, 2012, 1722-1730, ISSN 1026-3098, http://doi.org/10.1016/j.scient.2012.10.031.

[7] Shekar, B. H.; Bharathi, P.: Discrete Cosine Transformation and Height Functions Based Shape Representation and Classification, Procedia Computer Science, Volume 58, 2015, 714-722, ISSN 1877-0509, http://doi.org/10.1016/j.procs.2015.08.092.

[8] Wang, Junwei; Bai, Xiang; You, Xinge; Liu, Wenyu; Latecki, Longin Jan: Shape matching and classification using height functions. Pattern Recognition Letters, 33, 134-143, 2012 http://doi.org/10.1016/j.patrec.2011.09.042.

[9] Yang, Mingqiang; Kpalma, Kidiyo; Ronsin, Joseph: A Survey of Shape Feature Extraction Techniques, Pattern Recognition Techniques, Technology and Applications, Peng-Yeng Yin, IntechOpen, 2008, http://doi.org/10.5772/6237. 